\title{
Dieta del cernícalo americano (Falco sparverius Linnaeus, 1758) en dos localidades del valle interandino del norte de Ecuador
}

\section{Diet of American Kestrel (Falco sparverius Linnaeus, 1758) in two localities at the north ecua- dorian interandean valley}

\section{Glenda M. Pozo-Zamora ${ }^{1 *}$, Jonathan Aguirre ${ }^{1}$, Jorge Brito ${ }^{1,2}$}

\author{
1 Instituto Nacional de Biodiversidad, Calle Rumipamba 341 y Av. de los Shyris, Casilla: 17-07-8976, Quito, Ecuador \\ 2 Instituto de Ciencias Biológicas, Escuela Politécnica Nacional, Calle Ladrón de Guevara, Casilla: 17-01- 2759, Quito, Ecuador. \\ *Autor para correspondencia: \\ Email Glenda M. Pozo-Zamora: glenda.pozo@yahoo.es \\ Email Jonathan Aguirre: jonathan_mauricio18@hotmail.com \\ Email Jorge Brito: jorgeyakuma@yahoo.es
}

\begin{abstract}
Resumen
Damos a conocer la dieta del Cernícalo Americano Falco sparverius, por medio del análisis de 205 egagrópilas provenientes de dos localidades en la región norte interandina de Ecuador. Los ítems presa más representativos de Sangolquí y Tababela fueron los coleópteros (48.4 y 39.5\%), seguidos por ortópteros (31.3 y $30.7 \%$ ) respectivamente. En términos de biomasa, los mamíferos fueron el grupo de mayor importancia alimenticia en ambos sitios; siendo el roedor invasor Mus musculus la presa de preferencia; es decir que ésta rapaz estaría brindando un apreciable servicio como controlador de plagas. El índice de Shannon $\left(H^{\prime}=1.894\right)$ indica una mediana diversidad de presas y la amplitud de dieta $(0.26)$ sugiere que Falco sparverius presenta una dieta especialista en los dos sitios. Difiriendo de otros estudios donde presenta una dieta generalista.
\end{abstract}

Palabras clave: Alimentación; ecología trófica; egagrópilas; presas; Pichincha

\section{Abstract}

We present the diet of American Kestrel Falco sparverius, through pellets analysis, from two different localities in the Ecuadorian north-interandean region. The most representative prey items from Sangolquí and Tababela were Coleoptera (48.4, and $39.5 \%$ ), followed by Orthoptera (31.3 and $30.7 \%$ ), respectively. In terms of biomass, mammals were the most important food resource in both sites, the invasive rodent Mus musculus was the most preferred prey; it would provide an appreciable predatory as pest control service. The Shannon index $\left(H^{\prime}=1.894\right)$ indicates a median diversity of prey and, a diet amplitude of 0.26 suggests that the American Kestrel in our study sites is a specialist. Its diet differs from its relatives from other habitats where they present a generalist diet.

Keywords: feeding; pellets; Pichincha; preys; trophic ecology

\section{Citación:}

Pozo-Zamora G.M., J. Aguirre, J. Brito. 2017. Dieta del cernícalo americano (Falco sparverius Linnaeus, 1758) en dos localidades de valle interandino del norte de Ecuador. Revista peruana de biología 24(2): 145 - 150 (Julio 2017). doi: http://dx.doi.org/10.15381/rpb.v24i2.12305

\section{Permisos de colecta:}

El Ministerio del Ambiente de Pichincha Ecuador facilitó el permiso de investigación No. 01-2014-RIC-FAU-FLO-DPAP-MA.

$\begin{array}{ll}\text { Presentado: } & 07 / 08 / 2016 \\ \text { Aceptado: } & 10 / 05 / 2017\end{array}$

Aceptado: $\quad 10 / 05 / 2017$

Publicado online: $20 / 07 / 2017$
Información sobre los autores:

GMPZ y JB concibieron el estudio. GMPZ identificó muestras de aves, realizó la interpretación de datos y escribió el primer borrador del manuscrito. JA identificó las muestras de invertebrados y revisó el manuscrito. JB identificó muestras de mamíferos y reptiles, analizó los datos y realizo revisión crítica del manuscrito. Todos los autores aprobaron la versión final del manuscrito.

Los autores no incurren en conflicto de intereses. 


\section{Introducción}

El Cernícalo Americano (Falco sparverius) es una rapaz de tamaño pequeño, ampliamente distribuida en el continente americano, extendida desde Alaska hasta Tierra de Fuego en Argentina, contando con 17 subespecies a lo largo de su distribución (White et al. 2016). Aspectos alimenticios de $F$. sparverius han sido estudiados tanto en Norte y Centroamérica (Heintzelman 1964, Jenkins 1970, Cruz 1976, Hiraldo et al. 1991), así como en Sudamérica, donde se han desarrollado estudios de historia natural en Venezuela (Balgooyen 1989); mientras análisis de egagrópilas, restos de presas y avistamientos de alimentación han sido efectuados en Chile (Mella 2002, Figueroa \& Corales 2002, Figueroa \& Corales 2004, Rodríguez-San Pedro \& Allendes 2015), Argentina (Sarasola et al. 2003, Liébana et al. 2009, Santillan et al. 2009, Pagnoni 2013) y Brasil (Cabral et al. 2006, Zilio 2006, Aguiar et al. 2012).

Varios estudios demuestran que Falco sparverius es una rapaz generalista, que se alimenta de un espectro bastante variado de presas, siendo los artrópodos (principalmente insectos) los que predominan en relación a los vertebrados (Heintzelman 1964, Jenkins 1970, Cruz 1976, Jaksic et al. 1997, Sarasola et al. 2003).
En Ecuador únicamente han sido reportados registros visuales de las presas cazadas por Falco sparverius (Salazar et al. 2012, Ramírez-Jaramillo 2015), sin contar con información detallada del análisis de su dieta. El presente trabajo constituye el primer esfuerzo para documentar la dieta de Falco sparverius en Ecuador, basado en el análisis de egagrópilas en dos localidades de la provincia de Pichincha. Con este trabajo pretendemos contribuir al conocimiento de la ecología trófica de esta rapaz, lo cual es fundamental para comprender las estrategias de alimentación y dinámica de esta especie con el ecosistema.

\section{Material y métodos}

Entre septiembre del 2013 y septiembre del 2015, colectamos egagrópilas en dos localidades en la provincia de Pichincha (ubicadas en el piso Zoogeográfico Templado; Albuja et al. 2012) durante visitas esporádicas a dos localidades (Tabla 1): (1) casa antigua Caraburo, adyacente al Aeropuerto Internacional Mariscal Sucre en la parroquia Tababela $\left(0^{\circ} 07^{\prime} 51.11^{\prime \prime}\right.$, $78^{\circ} 21^{\prime} 6.31^{\prime \prime} \mathrm{W}, 2371 \mathrm{~m}$ ) allí predominan arbustos nativos como Acacia sp., Opuntia sp. y Croton sp., donde registramos a una familia de Falco sparverius (Fig. 1); y (2) Sangolquí, zona urbana ubicada al sureste de la ciudad de Quito (0¹9’34.19”'S,

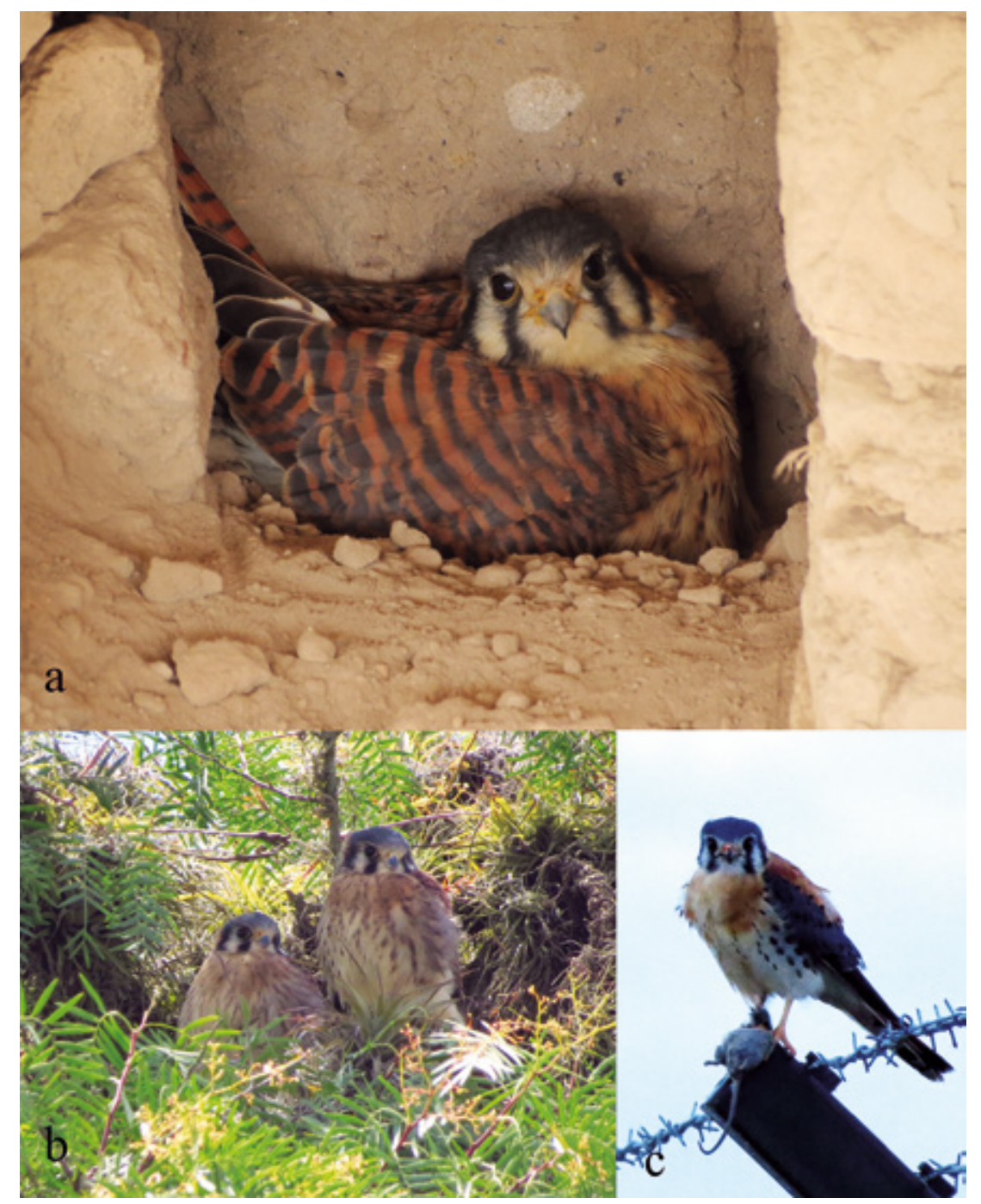

Figura 1. Individuos de Falco sparverius fotografiados en la localidad Tababela, donde fueron colectadas egagrópilas: a) Hembra anidando en pared de casa abandonada, b) jóvenes perchados en vegetación adyacente a casa, c) macho adulto acabando de cazar un roedor Phyllotis haggardi. 
Tabla 1. Fechas y número de egagrópilas colectadas en las dos localidades del valle interandino del norte de Ecuador.

\begin{tabular}{cccc}
\hline & & Sangolquí & Tababela \\
\hline \multirow{2}{*}{2013} & Sep & 12 & - \\
& Oct & 53 & - \\
& Nov & 14 & - \\
2014 & Ene & 10 & - \\
$\mathbf{2 0 1 5}$ & Sep & - & 116 \\
\hline Total & & $\mathbf{8 9}$ & $\mathbf{1 1 6}$ \\
\hline
\end{tabular}

78²7’30.38”W, $2492 \mathrm{~m}$ ), donde se observó el dormidero de una hembra de $F$. sparverius.

En total recolectamos 205 egagrópilas: 116 en Tababela y 89 en Salgolquí. Secamos las egagrópilas a temperatura ambiente y tomamos medidas de longitud y ancho (en $\mathrm{mm}$ ) utilizando un calibrador digital Buffalo Tools, con precisión de $\pm 0.01 \mathrm{~mm}$. Pesamos cada egagrópila en una balanza analítica Fisher Scientific $120 \mathrm{~g} \times 0.001 \mathrm{~g}$. El contenido de las egagrópilas fue separado, etiquetado con códigos únicos y se depositó en la colección del Museo Ecuatoriano de Ciencias Naturales del Instituto Nacional de Biodiversidad (MECN), Quito.

Para la identificación y peso promedio de los ítems presa, se recurrió a estudios y claves disponibles en mamíferos (e.g. Tirira 2007, Weskler \& Percequillo 2011), artrópodos (Roth 1973, Toro et al. 2003, Triplehorn \& Johnson 2005, Foottit \& Adler 2009, Cadena et al. 2016) y reptiles (Ramírez-Jaramillo et al. 2015), también comparamos con especímenes de la colección de fauna del (MECN).

El Número Mínimo de Individuos (NMI) en vertebrados y artrópodos, se determinó contando el número de mandíbulas homólogas o restos de cráneos, no se empleó otras partes del esqueleto con el fin de evitar reconteo (Manning \& Jones 1990). La biomasa fue obtenida multiplicando el peso promedio de las especies (en gramos) por su NMI (Herrera \& Jaksic 1980).

Para obtener la diversidad de presas en la dieta de Falco sparverius aplicamos el Índice de Shannon-Wiener (Moreno 2001):

$$
\mathrm{H}^{\prime}=-\sum p_{i}{ }^{*}\left(\ln p_{i}\right)
$$

Para medir el grado de semejanza de la dieta entre las dos localidades, usamos el Índice de Similitud Morisita-Horn:

$$
\mathrm{I}_{\mathrm{M}-\mathrm{H}}=\frac{2 \sum\left(a n_{i}^{*} b n_{j}\right)}{(d a+d b)^{*} a N * b}
$$

Utilizando el programa EstimateS, Versión 9 (Colwell 2013). Para determinar la amplitud del nicho trófico, aplicamos el Índice estandarizado de Levins (Levins 1968):

$$
B=1 \frac{1}{\sum p_{j}^{2}}
$$

que expresa el uso observado por el cernícalo desde 0 hasta $n$ presas, es decir distingue si existe especialización en la dieta (Krebs 1999, Rau 2000), para lo cual usamos el programa HaviStat 2.2 (Montenegro et al. 2014).

\section{Resultados}

Analizamos un total de 205 egagrópilas, que presentaron forma redondeada ligeramente triangulares y de coloración marrón. Las egagrópilas de Sangolquí $(n=89)$ presentaron las siguientes medidas $(\mathrm{mm})$ : largo $7.8-38.6(18.9 \pm 3.8)$, ancho $8.1-15.6(11.1 \pm 1)$, peso $0.1-0.9(0.4 \pm 0.1 \mathrm{~g})$ y número de presas por egagrópila $1-4(2.1 \pm 1.1)$; mientras que los regurgitos de Tababela $(\mathrm{n}=116)$ midieron $(\mathrm{mm})$ : largo $10.2-34.3(20.4 \pm 4.4)$, ancho $7.3-16.8(11.8 \pm 1.7)$, peso $0.1-0.7(0.4 \pm 0.1 \mathrm{~g})$ y número de presas por egagrópila $1-4$ $(2.1 \pm 1)$. Las egagrópilas de Falco sparverius en ambos sitios presentaron similar tamaño y peso.

En los 205 regurgitos analizados, registramos 786 presas (463 para Sangolquí y 323 para Tababela) agrupados en 20 ítems (13 en Sangolquí y 18 en Tababela). En la localidad de Sangolquí, los invertebrados fueron el grupo más frecuente con $96.4 \%$, donde los coleópteros representaron el 48.4\%, seguido por los ortópteros con el 31.3\%, los arácnidos con el 13.2\% y los escorpiónidos con el 3.5\%. Mientras que los vertebrados fueron registrados en una proporción de 3.7\% (Fig. 2). En la localidad de Tababela también se registró como ítem presa más frecuente a los invertebrados con una representatividad del $86.3 \%$, donde los coleópteros representaron el 39.5\%, los ortópteros el $30.7 \%$, los arácnidos $10.5 \%$ y los escorpiónidos con el $5.6 \%$. Los vertebrados se registraron en una proporción de $13.5 \%$, donde los mamíferos fueron los mejor representados con el 9.8\%, entre ellos el roedor introducido Mus musculus presentó una representatividad del 7.7\% (Tabla 2).

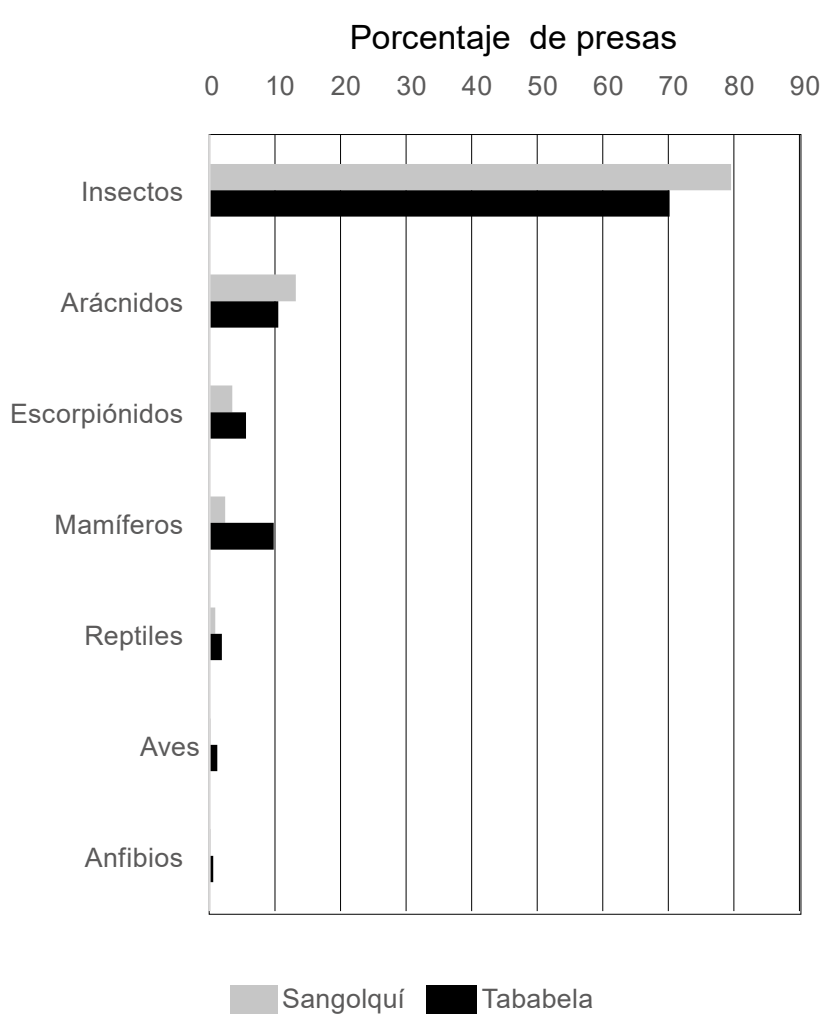

Figura 2. Comparación de la dieta de Falco sparverius, según el NMI (Número mínimo de individuos) en las dos localidades en el norte de Ecuador. 
Tabla 2. Composición de la dieta de Falco sparverius en las dos localidades del norte de Ecuador.

\begin{tabular}{|c|c|c|c|c|c|}
\hline \multirow{2}{*}{ Orden / Familia / Especie } & \multirow{2}{*}{ Peso } & \multicolumn{2}{|c|}{ Sangolquí } & \multicolumn{2}{|c|}{ Tababela } \\
\hline & & Total (\%) & BM (\%) & Total (\%) & BM (\%) \\
\hline MAMMALIA & & $11(2.4)$ & $155(40.3)$ & $34(9.8)$ & $465(70.8)$ \\
\hline \multicolumn{6}{|l|}{ Rodentia } \\
\hline \multicolumn{6}{|l|}{ Cricetidae } \\
\hline Akodon cf, mollis & 15 & $1(0.2)$ & $15(3.9)$ & $2(0.6)$ & $30(4.6)$ \\
\hline Phyllotis haggardi & 20 & & & $2(0.6)$ & $40(6.1)$ \\
\hline Reithrodontomys soderstromi & 15 & & & $3(0.9)$ & $45(6.8)$ \\
\hline \multicolumn{6}{|l|}{ Muridae } \\
\hline Mus musculus & 14 & $10(2.2)$ & $140(36.4)$ & $25(7.7)$ & $350(53.3)$ \\
\hline \multicolumn{6}{|l|}{ REPTILES } \\
\hline \multicolumn{6}{|l|}{ Squamata } \\
\hline \multicolumn{6}{|l|}{ Iguanidae: Tropidurinae } \\
\hline Stenocercus guentheri & 11 & $4(0.9)$ & $44(11.4)$ & $6(1.9)$ & $66(10)$ \\
\hline \multicolumn{6}{|l|}{ AMPHIBIA } \\
\hline \multicolumn{6}{|l|}{ Anura } \\
\hline \multicolumn{6}{|l|}{ Craugastoridae } \\
\hline Pristimantis unistrigatus & 3 & $1(0.2)$ & $3(0.8)$ & $2(0.6)$ & $6(0.9)$ \\
\hline \multicolumn{6}{|l|}{ AVES } \\
\hline \multicolumn{6}{|l|}{ Paseriformes } \\
\hline Phrygilus plebejus & 15 & $1(0.2)$ & $15(3.9)$ & $4(1.2)$ & $60(9.6)$ \\
\hline \multicolumn{6}{|l|}{ INVERTEBRADOS } \\
\hline Coleoptera & & $224(48.4)$ & $135.3(35.2)$ & $128(39.5)$ & $34(5.5)$ \\
\hline \multicolumn{6}{|l|}{ Carabidae } \\
\hline Anchomenus aff, quitensis & 0.15 & & & $3(0.9)$ & $0,4(0.1)$ \\
\hline Cerambicidae & 0.19 & & & $51(15.8)$ & $9.7(1.5)$ \\
\hline Curculionidae & 0.92 & $124(26.7)$ & $114(29.6)$ & & \\
\hline Chrysomelidae & & & & $2(0.6)$ & \\
\hline \multicolumn{6}{|l|}{ Elateridae } \\
\hline Chalcolepidius sp & 0.13 & & & $1(0.3)$ & $0.13(0)$ \\
\hline \multicolumn{6}{|l|}{ Melolonthidae } \\
\hline Barotheus andinus & 0.51 & $2(0.4)$ & $1(0.3)$ & & \\
\hline Clavipalpus whymperi & 0.3 & $4(0.9)$ & $1.2(0.3)$ & $3(0.9)$ & $0,9(0.1)$ \\
\hline Heterogomphus bourcieri & 1 & $7(1.5)$ & & $10(3.1)$ & $10(1.5)$ \\
\hline Platycoelia cf, lutescens & 0.4 & & & $1(0.3)$ & $0.4(0.4)$ \\
\hline Coleopteros Indeterminados & 0.22 & $87(18.8)$ & $19.1(5)$ & $57(17.6)$ & $12.5(1.9)$ \\
\hline \multicolumn{6}{|l|}{ Orthoptera } \\
\hline \multicolumn{6}{|l|}{ Acrididae } \\
\hline Paradichroplus sp & 0.16 & $145(31.3)$ & $23.2(6)$ & $99(30.7)$ & $15.8(2.4)$ \\
\hline \multicolumn{6}{|l|}{ Aranea } \\
\hline Lycosidae & & $61(13.2)$ & & $34(10.5)$ & \\
\hline \multicolumn{6}{|l|}{ Scorpionida } \\
\hline \multicolumn{6}{|l|}{ Chactoidae } \\
\hline Teuthraustes atramentarius & 0.57 & $16(3.5)$ & $9.1(2.4)$ & $18(5.6)$ & $10.2(1.6)$ \\
\hline Total individuos & & $463(100)$ & $384.6(100)$ & $323(100)$ & $657.22(100)$ \\
\hline
\end{tabular}


En cuanto a la biomasa, en Sangolquí los mamíferos aportaron con el $40.3 \%$ seguido por los coleópteros con $35.2 \%$ y los reptiles con el $11.4 \%$, los otros grupos presentaron representatividad menor. En Tababela los mamíferos presentaron el principal aporte de biomasa, con el $70.8 \%$, seguido por los reptiles con el $10 \%$ y las aves $9.6 \%$, los otros grupos presentaron representatividad menor (Fig. 3).

El valor de la amplitud de la dieta del cernícalo fue de 0.20 para Sangolquí y 0.27 para Tababela, y en conjunto 0.26 , lo cual refleja una baja amplitud de presas sugiriendo una dieta especialista. El Índice Shannon-Wiener para Sangolquí $\left(\mathrm{H}^{\prime}=\right.$ $1.705)$, para Tababela $\left(H^{\prime}=2.084\right)$ y en conjunto $\left(H^{\prime}=1.894\right)$, nos indican una mediana diversidad de presas. Finalmente, la similitud entre las localidades Sangolquí y Tababela respecto a los ítems presa consumidos por Falco sparverius es de $74.2 \%$.

\section{Discusión}

Numéricamente los invertebrados presentaron la mayor parte de la dieta de Falco sparverius (92.2\%) en el norte de Ecuador (Tabla 2), ratificando los resultados previamente reportados en Sudamérica (Balgooyen 1989, Mella 2002, Sarasola et al. 2003, Figueroa \& Corales 2004, Cabral et al. 2006, Zilio 2006, Liébana et al. 2009, Santillán et al. 2009) donde se cataloga a esta rapaz como primariamente insectívoro. Sin embargo, nuestro estudio difiere del efectuado por Figueroa y Corales (2002), en época de invierno en latitudes australes, y del estudio de Heintzelman (1964), realizado en Pennsylvania, EE.UU, donde señalan que la mayoría de presas son vertebrados, principalmente roedores. Esta variación podría estar ligada a la estacionalidad y condiciones ecológicas, favoreciendo mayor disponibilidad de recurso de vertebrados y la posible escasez de invertebrados en épocas invernales (Figueroa \& Corales 2002).

En términos de biomasa, en nuestro estudio los mamíferos son los más importantes dentro de la dieta del cernícalo americano con el 59.5\%, concordando con estudios anteriores donde se indica que el mayor aporte de biomasa proviene de los vertebrados (Cruz 1976, Balgooyen 1989, Sarasola et al. 2003, Figueroa \& Corales 2002, Figueroa \& Corales 2004). Es meritorio destacar que dentro de los mamíferos, el roedor invasor Mus musculus fue la presa de preferencia de Falco sparverius; es decir que ésta rapaz estaría brindando un apreciable servicio como controlador de plagas.

Existen observaciones de individuos de F. sparverius cazando murciélagos a lo largo del continente americano (James \& Hayse 1963, Aguiar et al. 2012, Pagnoni 2013, Lenoble et al. 2014, Rodríguez-San Pedro \& Allendes 2015, Martínez et al. 2016) y observaciones en Ecuador (Salazar et al. 2012) cazando serpientes (Colubridae), aunque éstos ítems no han sido reportados en egagrópilas; sin embargo, con el desarrollo de futuros estudios en otras localidades, será posible esclarecer los límites y preferencias alimenticias de éste falconiforme.

El Índice de Shannon nos dio un valor medio, mostrando una mediana diversidad de presas en la dieta de Falco sparverius en las dos localidades de la provincia de Pichincha. Mientras que la amplitud de dieta resultó en un valor bajo, sugiriendo que ésta especie presenta una dieta relativamente especialista. Esta especialización podría estar influenciada por la disponibilidad del recurso alimenticio, mostrando más bien un comportamiento oportunista, siendo ésta una respuesta funcional a la disponibi- lidad de presas (Farias \& Jaksic 2007a, 2007b). Ésta tendencia coincide con lo mencionado por Cabral et al. (2006) para la dieta de F. sparverius en Brasil.

\section{Agradecimientos}

N. Zamora y A. Pozo permitieron la recolección de egagrópilas en su propiedad, R. Calderón de Corporación Quiport facilitó las colectas en Tababela. J. Curay y R. Vargas asistieron en el trabajo de laboratorio (MECN-INB). M. Sánchez revisó el Abstract.

\section{Literatura citada}

Aguiar L.M.S., A. Motta \& C. Esberárd. 2012. Falco sparverius (Aves: Falconiformes) preying upon Nyctinomops laticaudatus (Chiroptera: Molossidae). Zoologia 29(2):180-182. doi: http://dx.doi.org/10.1590/S1984-46702012000200011

Albuja L., A. Almendáriz, R. Barriga, L.D. Montalvo, F. Cáceres \& J.L. Román. 2012. Fauna de Vertebrados del Ecuador. Quito: Instituto de Ciencias Biológicas, Escuela Politécnica Nacional.

Balgooyen T.G. 1989. Natural history of the American Kestrel in Venezuela. Journal Raptor Research 23(3):85-93. https:// sora.unm.edu/node/53138

Cabral J.C., M.A. Monteiro \& J.C. Motta-Junior. 2006. Dieta do quiriquiri, Falco sparverius (Aves: Falconiformes), na Estação Ecológica de Itirapina, SP. Revista Brasileira de Ornitología 14(4):393-399.

Cadena-Ortíz H, Garzón C, Villamarín-Cortéz S, et al. 2016. Diet of the Burrowing Owl Athene cunicularia, in two locations of the inter-Andean valley Ecuador. Brazilian Journal of Ornithology 24(2):122-128.

Colwell R. K. 2013. EstimateS: Statistical estimation of species richness and shared species from samples. Version 9. User's Guide and application published at: http://purl.oclc.org/estimates.

Cruz A. 1976. Food and foraging ecology of the American Kestrel in Jamaica. Condor 78:409-412. https://sora.unm.edu/ node/ 102653

Farias A.A. \& F.M. Jaksic. 2007a. El Niño events, the lean versus fat scenario, and long-term guild dynamics of vertebrate predators in a South American semiarid ecosystem. Austral Ecology 32:225-238. doi: http://dx.doi.org/10.1111/j.14429993.2007.01684.x

Farias A.A. \& F.M. Jaksic. 2007b. Effects of functional constraints and opportunism on the functional structure of a vertebrate predator assemblage. Journal of Animal Ecology 76:246-257. doi: http://dx.doi.org/10.1111/j.1365-2656.2006.01202.x

Figueroa R.A. \& E.S. Corales. 2002. Winter diet of the American Kestrel (Falco sparverius) in the Forested Chilean Patagonia, and its relation to the availability of prey. International Hawkwatcher 5:7-14.

Figueroa R.A. \& E.S. Corales. 2004. Summer diet comparison between the American Kestrel (Falco sparverius) and Aplomado Falcon (Falco femoralis) in an agricultural area of Araucanía, southern Chile. Hornero 19(2):53-60. http://www.scielo. org.ar/pdf/hornero/v19n2/v19n2a02.pdf

Foottit R. \& P. Adler. 2009. Insect Biodiversity: Science and Society. Blackwell Publishing. Hoboken, New Jersey.

Heintzelman D.S. 1964. Spring and summer Sparrow Hawk food habitats. Wilson Bulletin 76:323-330. https://sora.unm. edu/node/128169

Herrera C.M. \& F.M. Jaksic. 1980. Feeding ecology of the barn owl in Central Chile and southern Spain: a comparative study. The Auk 97:760-767.http://www.jstor.org/ stable/4085747?seq=1\#page_scan_tab_contents

Hiraldo F., M. Delibes, J. Bustamante \& R.R. Estrella. 1991. Overlap in the Diets of Diurnal Raptors Breeding at the Michilía Biosphere Reserve, Durango, Mexico. Journal Raptor Research 25(2):25-29. https://sora.unm.edu/node/53211

Jaksic F. M., P. Feinsinger \& J.E. Jimenez. 1993. A long-term study on the dynamics of guild structure among predatory vertebrates at a semi-arid Neotropical site. Oikos 67: 87-96.

Jaksic F.M, S.I. Silva, P.L. Meserve \& R.J. Gutierrez. 1997. A long-term study of vertebrate predator responses to an El Niño (ENSO) 
disturbance in western South America. Oikos 78:341-354. doi: http://dx.doi.org/10.2307/3546302

Jenkins R.E. 1970. Food Habits of Wintering Sparrow Hawks in Costa Rica. The Wilson Bulletin 82(1):97-98. https://sora.unm. edu/node/ 128647

James P. \& A. Hayse. 1963. Sparrow Hawk preys on Mexican FreeTailed bat at Falcon Reservoir. Journal of Mammalogy 44(4):574-575. doi: http://dx.doi.org/10.2307/1377142

Krebs C. J. 1999. Ecological methodology. 2 ed. Menlo Park, California: Benjamin/Cummings.

Lenoble A., C. Bochaton, T. Bos, E. Discamps \& A. Queffelec. 2014. Predation of Lesser Naked-Backed bats (Pteronotus davyi) by a pair of American Kestrels (Falco sparverius) on the Island of Marie-Galante, French West Indies. Journal Raptor Research 48(1):78-81. doi: http://dx.doi.org/10.3356/JRR-13-28.1

Levins R. 1968. Evolution in changing environments. Princeton Univ. press, Princeton, N. J. $120 \mathrm{pp}$.

Liébana M.S., J.H. Sarasola \& M.S. Bó. 2009. Parental care and behavior of breeding American Kestrels (Falco sparverius) in Central Argentina. Journal Raptor Research 43(4):338-344. doi: http://dx.doi.org/10.3356/JRR-08-82.1

Manning R.W. \& J.K. Jones. 1990. Remains of small mammals recovered from Barn Owl pellets from Crosby county, Texas. Texas Journal of Science 42:311-312.

Martínez T.A., M.J. Rodríguez-Muñoz, F.D. Maidana, G.A. Fava1, J.C. Acosta \& G.M. Blanco. 2016. Halconcito colorado (Falco sparverius) depredando murciélago en la ciudad de Mendoza, Argentina. Nuestras Aves 61:19.

Mella J. E. 2002. Dieta del Cernícalo (Falco sparverius) y del Tucúreque (Bubo magellanicus) en un ambiente cordillerano de Chile Central. Boletín Chileno de Ornitología 9:34-37.

Montenegro J.A., A. Acosta \& J.D. Reimer. 2014. HaviStat@ v2.2: Application to estimate preference for habitat and resources. Universitas Scientarium 19(3):333-337. doi: http://dx.doi. org/10.11144/Javeriana.SC19-3.haea

Moreno C. E. 2001. Métodos para medir la biodiversidad. M\&TManuales y Tesis SEA, vol. 1. Zaragoza, $84 \mathrm{pp}$.

Pagnoni G. 2013. Halconcito Colorado (Falco sparverius cinnamominus) cazando murciélagos. Nuestras aves 58:59-60.

Ramírez-Jaramillo S. M. 2015. Predación de Falco sparverius en la localidad de Juan Montalvo, Carchi-Ecuador. Boletín Técnico, Serie Zoológica 12(10-11): 1-10. http://ugi.espe.edu. ec/ugi/wp-content/uploads/2013/10/OPT-Boletin-Tec.12-Serie-Zoologica.pdf

Ramírez-Jaramillo S., P. Bejarano-Muñoz, M. Rodríguez-Badillo \& M. Yánez-Muñoz. 2015. Uso de perchas nocturnas por Stenocercus guentheri (Iguanidae:Tropidurinae) en dos ecosistemas del distrito metropolitano de Quito (Ecuador). Boletín de la Asociación Herpetológica Española 26(1):29-32. https:// dialnet.unirioja.es/servlet/articulo?codigo $=5503159$
Rau J. 2000. Métodos de ecología trófica. Pp. 397-406, en: Mamíferos de Chile (A Muñoz Pedreros y J Yáñez, eds.). CEA Ediciones, Valdivia, Chile.

Rodríguez-San Pedro A. \& J.L. Allendes. 2015. Depredación del murciélago de cola libre Tadarida brasiliensis (I. Geoffroy Saint Hilaire, 1824) por el cernícalo Falco sparverius Linnaeus, 1758, en un sector urbano de Santiago, Región Metropolitana, Chile. Biodiversity and Natural History 1:6-8.

Roth M. 1973. Sistemática y Biología de los Insectos. ParaninfoMadrid. $151 \mathrm{pp}$.

Salazar M.V., H.F. Cadena \& E. Bonnacorso. 2012. Desarrollo de los polluelos y cuidado parental en el Quilico (Falco sparverius) en el suroeste del Ecuador. Boletín SAO Revista científica de la Sociedad Antioqueña de Ornitología 20(2):61-66. http://sao.org.co/publicaciones/boletinsao/20\% $282 \% 29 / 20 \% 282 \% 29 . h t m l$

Santillán M.A., A. Travaini, S.C. Zapata, A. Rodríguez, J.A. Donázar, D.E. Procopio \& J.I. Zanón. 2009. Diet of the American Kestrel in Argentine Patagonia. Journal Raptor Research 43(4):377-381. doi: http://dx.doi.org/10.3356/JRR-0880.1

Sarasola J.H., M.A. Santillán \& M.A. Galmes. 2003. Food habits and foraging ecology of American Kestrels in the semiarid forest of Central Argentina. Journal Raptor Research 37(3):236243. https://sora.unm.edu/node/54046

Tirira D. 2007. Guía de Campo de los Mamíferos del Ecuador. Publicación especial sobre los mamíferos del Ecuador. 6. Ediciones Murciélago Blanco, Quito, Ecuador.

Toro H., E. Chiappa \& C. Tobar. 2003. Biología de Insectos. Ediciones Universitarias de Valparaiso. Chile.

Triplehorn CH. \& N. Johnson. 2005. Borror and Delong's Introduction to the Study of Insects. Thomson Brooks/Cole. Seven Edition. United States of América.

Weskler M. \& A.R. Percequillo. 2011. Key to the genera of the tribe Oryzomyini (Rodentia: Cricetidae: Sigmodontinae). Mastozoología Neotropical 18(2):281-292. https://www.sarem. org.ar/mastozoologia-neotropical-vol18-no2/

White C.M., G.M. Kirwan, D.A. Christie \& P. Boesman. 2016. American Kestrel (Falco sparverius), en: J. del Hoyo, A. Elliott, J. Sargatal, D.A. Christie, E. de Juana (Eds.), Handbook of the Birds of the World Alive. Lynx Edicions, Barcelona. Enlace: http://www.hbw.com/node/53219. Acceso 14/03/2016.

Zilio F. 2006. Dieta de Falco sparverius (Aves:Falconidae) e Athene cunicularia (Aves:Strigidae) em uma regiáo de dunas no sul do Brasil. Revista Brasileira de Ornitologia 14(4):379-392. 